\title{
Uma Arquitetura Fog-Cloud para o Monitoramento de Sinais Corporais
}

\author{
Wagno Sérgio Leão, Gabriel Di iorio Silva, Victor Ströele, Mário Dantas \\ Institute of Exact Sciences - Federal University of Juiz de Fora (UFJF) \\ Rua José Lourenço Kelmer - São Pedro, Juiz de Fora - MG, 36036-900 - Brazil \\ Departamento de Ciência da Computação \\ \{wagno.leao, iorio, victor.stroele, mario.dantas\}aice.ufje.br
}

\begin{abstract}
Com a popularização de sensores vestíveis cada vez mais disseminada, a observação de dados corporais se tornou ainda mais pertinente. Por meio do estudo dos dados fornecidos por tais sensores, juntamente com dados de localização, para enriquecer as análises, somos capazes de detectar situações de estresse. Para que tal tarefa seja exequível, é necessária uma arquitetura robusta que comporte o volume de dados a serem consumidos pelos sistemas que detectam tais situações. Dessa forma, este trabalho apresenta uma proposta de arquitetura Fog-Cloud que faz uso de dados corporais e de localização por meio de técnicas de Machine Learning para determinar momentos de estresse do usuário.
\end{abstract}

\section{Introdução}

Ferramentas para monitoramento de usuários tem se tornado cada vez mais populares no cotidiano dos cidadãos [Bove 2019]. Equipamentos que requisitavam um grande espaço físico agora podem ser carregados para todos os lugares nos pulsos e bolsos. Dessa forma, o monitoramento de diversas informações é facilitado. Graças ao avanço das tecnologias e, especialmente, ao fenômeno da Internet das Coisas (IoT) [Wan et al. 2018], agora as pessoas podem carregar esses aparelhos de forma prática e que são capazes de realizar diversas atividades, tais como: Medir a frequência cardíaca, oxigenação e pressão sanguínea juntamente com diversas outras informações.

Como resultado da popularização deste tipo de equipamento para os usuários, a análise e busca por uma melhor compreensão dos dados relacionados ao comportamento corporal se tornam, consequentemente, mais recorrentes [Bove 2019]. Assim, para performar tais análises, é importante destacar a grande importância da análise de dados e processos de Machine Learning para verificação de padrões e conclusões a respeito dos usuários que fazem uso dessas ferramentas. Além disso é possível também fazer contextualização dos dados corporais tendo como base a localização do usuário. Tal dado se mostra extremamente útil para uma análise mais detalhada quando levamos em consideração que o usuário pode apresentar padrões diferentes nos seus dados corporais dependendo da localização em que ele se encontra. A fim de realizar tal agregação, foi elaborada uma arquitetura que emprega a computação em Fog.

O ambiente de Cloud Computing possui como destaque principal a centralização de processos. Por outro, lado a Fog Computing tem o objetivo de oferecer um paradigma de descentralização tanto a nível rede quanto a nível computacional. A Fog Computing se apresenta como uma estrutura onde dados, computação e comunicação são distribuídos 
por todo o sistema de forma modular entre as fontes de dados e a nuvem. Desta forma, os dados recebidos pelo nó Fog podem ser pré-processados antes de serem enviados para a nuvem, reduzindo assim a latência da rede e evitando possíveis sobrecargas e atrasos da rede. Assim, esse paradigma é uma alternativa promissora para o desenvolvimento de sistemas de suporte e monitoramento em tempo real.

O artigo está organizado da seguinte forma: Na seção 2, são apresentados os trabalhos relacionados; Na seção 3, a proposta é discutida em detalhes; Na seção 4, são apresentados os resultados obtidos baseados na arquitetura Fog, além de discuti-los, baseados na arquitetura proposta. A seção 5 apresenta as considerações finais e trabalhos futuros.

\section{Trabalhos Relacionados}

Técnicas e ferramentas de monitoramento de pessoas por meio de dados coletados por sensores corporais têm sido objeto de diversas pesquisas. Isso pode ser verificado por meio de estudos sobre o estado da arte em sensores corporais, conforme mostrado em [Gravina and Fortino 2020]. Com o aumento do número de trabalhos que abordam essa temática, existem trabalhos que fazem uso dessas informações para identificar padrões de estresse e ansiedade [Ciabattoni et al. 2017]. Tais trabalhos são importantes para perceber algumas abordagens já tratadas em estudos anteriores e formas de buscar diferentes perspectivas para a solução do problema.

Além disso, também é interessante realizar uma análise das relações entre o pico de frequência cardíaca e o estresse. Para isso, estudos como [Santhanagopalan et al. 2018] foram de grande valia para compreender o comportamento dos dados corporais em situações de estresse e ansiedade para um melhor entendimento do problema a ser abordado e para o desenvolvimento do raciocínio que nortearam a construção da proposta. Para reconhecer o comportamento dos usuários no sistema proposto, os dados coletados devem ser processados por meio de técnicas de reconhecimento de padrões. Várias técnicas de aprendizado de máquina bem fundamentadas na literatura têm se mostrado eficazes. Algoritmos de classificação como K-Nearest Neighbor (KNN) e Support Vector Machines (SVM) são usados para identificar os padrões nos graus de estresse dos usuários [Aditya and Tibarewala 2012]. Da mesma forma, [Priya et al. 2020] faz uso de algoritmos de aprendizado de máquina para verificar níveis de intensidade diferentes de estresse.

Executar a coleta, processamento e visualização de dados de vários usuários simultaneamente não é uma tarefa trivial. Por este motivo, o sistema que é explicado neste artigo emprega uma operação baseada no paradigma Fog-Cloud [Munir et al. 2017] e usa estratégias e conceitos apresentados na arquitetura lambda [Kiran et al. 2015]. Tais ferramentas já provaram ser capazes de suportar a grande carga de dados recebidos dos usuários e não afetar o processamento e a entrega de informações. Em [Larcher et al. 2020], um framework para reconhecer padrões anômalos no comportamento de pessoas idosas foi implementada usando a arquitetura lambda para processamento em tempo real e o esquema Fog-Cloud para mitigar o volume de informações recebidas pela nuvem.

Logo, percebe-se que o diferencial dessa proposta encontra-se em fazer uso, na arquitetura, de dados de localização e de sensores corporais para gerar uma eficiência ainda maior no estudo de comportamento do usuário em questão tanto nas condutas de frequência quanto em sua rotina de deslocamento dentro do ambiente monitorado. 


\section{Arquitetura Proposta}

Este trabalho propõe a implementação de um sistema de monitoramento de usuários em tempo real por meio de sensores. A fim de desenvolver tal sistema, decidimos coletar seus dados em diferentes cenários e contextos. Sendo assim foram coletadas as informações relativas aos dados corporais juntamente com dados de localização para uma análise mais profunda do comportamento do usuário.

Levando em consideração o grande volume de dados gerados e a complexidade no fluxo computacional dessas informações, vimos vantagens em usar o paradigma FogCloud para coleta e processamento de dados, visando reduzir possíveis congestionamentos de rede, aproximar o processamento dos pontos de extração de dados e melhorar a capacidade de resposta geral do sistema.

Os dados gerados pelos sensores são produzidos em um dispositivo na Edge e enviados para um nó de processamento Fog. Além disso, a arquitetura do sistema também foi baseada nos conceitos da arquitetura Lambda, onde os dados recebidos são enviados simultaneamente para dois fluxos de processamento paralelos, onde tanto um processamento em batch quanto uma rotina de fluxo contínuo são implementadas de forma híbrida. Os resultados gerados então são enviados para a nuvem para que possam ser apresentados aos usuários finais através de ferramentas de monitoramento. A Figura 1 fornece uma visão geral da arquitetura proposta e suas camadas.

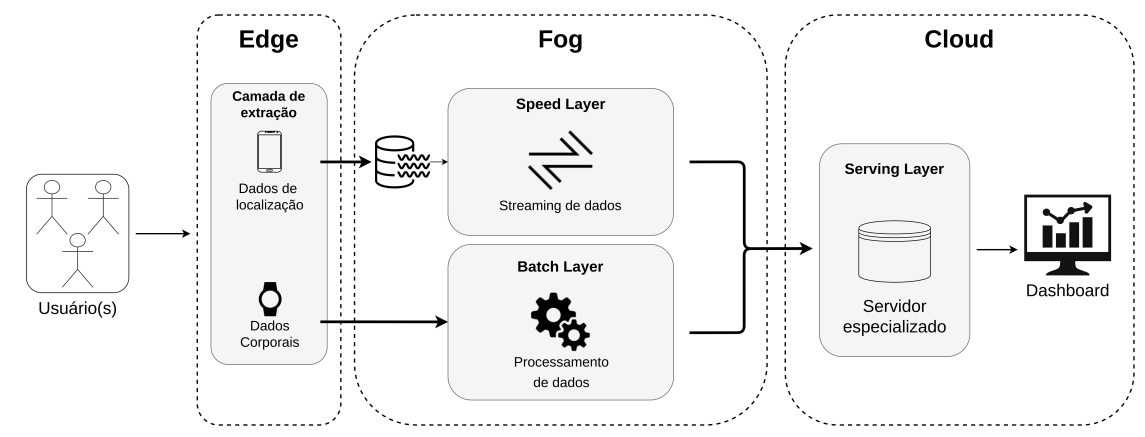

Figura 1. Arquitetura proposta com principais componentes e fluxo de dados.

O fluxo do sistema apresentado começa com a extração dos dados gerados pelos sensores de um ou mais usuários simultaneamente. Para isto, a camada de extração possui tal responsabilidade, sendo composta pelos sensores individuais de cada usuário que coletam as informações necessárias para o monitoramento. É importante ressaltar que, embora a coleta destes dados seja feita de forma contínua e em tempo real, o envio e processamento dos mesmos é feito de forma assíncrona, sendo apenas necessário que o momento em que a medição foi feita seja registrada. Após a coleta, os dados são enviados ao nó Fog para que possam ser pré-processados. No nó Fog, os dados corporais são armazenados junto aos dados de localização, que são armazenados em um banco de dados $S Q L$ que segue os mesmos conceitos de um Data Lake. Adotamos o uso de um Data Lake para possibilitar o armazenamento de um grande volume de dados a uma alta velocidade e também gerar disponibilidade contínua de seu conteúdo.

Ao realizar estudos de trabalhos similares, decidimos utilizar a arquitetura lambda porque ela apresenta resultados satisfatórios na solução de problemas envolvendo desempenho e modularidade. Como mencionado em [Kiran et al. 2015], a arquitetura lambda 
se concentra em oferecer alta escalabilidade e ter um baixo tempo de atraso tanto em consultas de dados quanto em atualizações. Ela implementa uma estrutura distribuída de três camadas principais: a batch layer, speed layer e a serving layer. Seguindo os princípios apresentados na arquitetura Lambda, a batch layer tem a função de realizar toda a computação de alto custo no sistema, de modo que os resultados gerados sejam armazenados e estejam sempre disponíveis para os outros componentes. Isto é feito desta forma pois garante que o sistema não seja prejudicado, por um possível atraso na resposta devido a sobrecargas de dados para serem processados. No sistema proposto, a batch layer recebe os dados corporais de um usuário específico e atualiza seu modelo de classificação através de técnicas de treinamento e avaliação de aprendizagem de máquina para gerar previsões cada vez mais precisas sobre o estado do indivíduo em um determinado contexto. Com a informação apropriada gerada, a mesma será armazenada em um banco de dados dedicado, e o modelo gerado usando os novos dados também será armazenado para garantir a tolerância do sistema a possíveis erros de classificação ou até mesmo falhas de hardware.

Ao mesmo tempo em que os novos dados estão sendo processados na batch layer, as mesmas informações também estão sendo enviadas para outro fluxo de trabalho, onde são usados na speed layer. Para garantir que o usuário-final tenha informações em tempo real, a speed layer é desenvolvida apenas com consultas de baixa latência e funções de processos de custo baixo, sendo que esta camada também pode ter a capacidade de usar os dados enriquecidos gerados pela batch layer para conseguir oferecer uma resposta adequada. A responsabilidade da speed layer no sistema proposto é assegurar o gerenciamento consistente do fluxo de dados, realizando também as transformações e validações necessárias para gerar as informações mais relevantes possíveis e auxiliar na tomada de decisão durante o monitoramento do comportamento dos indivíduos em um ambiente.

No final deste fluxo de processamento, os resultados gerados são enviados para a nuvem para serem utilizados pela serving layer. A principal tarefa da serving layer é disponibilizar as informações geradas e apresentá-las ao usuário final, além de assegurar que atualizações constantes dos dados sejam realizadas. Para monitorar e analisar os usuários, foi implementado um painel interativo com as visualizações mais relevantes dos dados, sendo que estas são atualizadas regularmente e podem ser acessadas remotamente por qualquer indivíduo com as credenciais permitidas.

\section{Avaliação da Arquitetura}

Para a realização do estudo proposto, devemos considerar diferentes possibilidades, devido à sua complexidade e abrangência no tratamento dos dados. Ao lidar especificamente com dados de sensores corporais, é preciso que estes sejam registrados tanto para análise quanto para a viabilidade de possíveis modelos e formas de se chegar a uma solução viável para o monitoramento de sinais corporais. Sendo assim, é necessário realizar um estudo meticuloso de cada um dos sinais capturados usados como variáveis de entrada para o modelo de aprendizado de máquina.

Porém, para enriquecer os dados do sensor corporal com informações sobre a localização do usuário em seu ambiente doméstico, devemos considerar alguns fatores. Primeiro, devemos estudar como coletar essas informações da maneira menos intrusiva. Além disso, a análise detalhada de possíveis interferências, formato dos dados, técnicas 
de armazenamento e discriminação do ambiente também é de suma importância para o desenvolvimento do trabalho de pesquisa. Assim, é possível perceber duas frentes de estudo significativas: (i) seu enriquecimento com dados obtidos na localização do usuário, e (ii) a coleta e análise de dados de sensores corporais. Para mais, é importante frisar que análises prévias com dados de frequência foram feitos por meio do simulador SIAFU que nos permitiu a realização de estudos iniciais com dados próximos dos que seriam vistos além de organizar uma forma de tratamento dos dados que possibilite sua operacionalização. É importante ressaltar, também, que os dados coletados nos experimentos realizados são de origem dos próprios autores, assegurando assim a aprovação do uso dessas informações.

\subsection{Estudo com Dados de Localização}

O sistema de localização proposto neste artigo foi implantado e testado por meio da plataforma Docker $^{1}$ em um servidor dedicado. A coleta de dados foi programada para funcionar em intervalos regulares de 1 minuto. Vale ressaltar que embora os testes de implantação tenham sido feitos em um único servidor, o desenvolvimento da arquitetura apresentada na Figura 1 foi feita através de conexões entre os Containers das aplicações usadas.

O sensor de localização foi implantado pela aplicação de smartphone (FIND $\left.3^{2}\right)$, enquanto para o sensor de dados corporais uma SmartBand foi usada, sendo estes sensores os elementos da camada de extração. A ferramenta de criação de fluxos de processos com dispositivos IoT Node-RED foi utilizada para o gerenciamento do fluxo dos dados recebidos e enviados, além do armazenamento das informações geradas, fazendo esta aplicação ser o componente central do processamento em Fog do sistema. Para a implantação do Data Lake, um banco de dados relacional SQLite3 foi usado e para o armazenamento das informações na serving layer foi utilizado o banco de dados relacional Postgres. As requisições dos dados armazenados no Data Lake são feitas através de uma Web API. Além disso, as visualizações das informações dos usuários foram criadas utilizando a ferramenta de análise e monitoramento Grafana. É importante enfatizar que os valores categóricos retornados pelo sensor precisam ser previamente especificados pelo usuário para que medições errôneas possam ser evitadas. Tal especificação é realizada através do treinamento de modelos de classificação, onde os dados das frequências de onda WiFi e Bluetooth são capturados pelo sensor para serem utilizados para o treinamento da área.

No total, quatro containers interconectados foram implantados para a criação do sistema: um banco de dados e a Web API das localizações medidas pelo FIND3, uma aplicação do Node-RED para o gerenciamento do fluxo de dados, um banco de dados Postgres e uma aplicação do Grafana para a geração do dashboard interativo. As variáveis utilizadas para monitorar a localização do usuário são os dados retornados do Data Lake, sendo que estas variáveis por si só se propõem serem suficientes para o monitoramento. No total, 112 entradas foram registradas no banco de dados da nuvem durante todo o experimento, onde 64 delas correspondem ao quarto do usuário, 23 à cozinha e 15 à sala de estar. As 10 entradas restantes correspondem a locais desconhecidos, os quais o usuário não especificou como uma região válida de monitoramento.O cenário desse experimento foi definido como sendo a casa de um dos usuário, onde os cômodos monitorados foram a sala, a cozinha e o quarto. O experimento teve duração de cerca de uma semana.

\footnotetext{
${ }^{1}$ https://www.docker.com/

${ }^{2}$ https://www.internalpositioning.com/doc/tracking_your_phone.md
} 


\subsection{Estudo com Sensores Corporais}

Para o acompanhamento comportamental de um dos autores e a análise de seu impacto na saúde, é necessário um estudo com dados abrangentes no aspecto temporal. Isso se mostra necessário pois devemos ter uma visão geral do comportamento por um período de tempo considerável e, desta forma, os dados não ficam a mercê de situações particulares. Portanto, foram considerados neste estudo os dados corporais de um dos autores referentes ao período de março de 2020 a março de 2021. É importante observar que alguns dados contêm valores faltantes devido à falta de uso da pulseira e problemas no envio dos dados. Assim, por se preocupar com a saúde do usuário em questão, nenhum tipo de abordagem para o preenchimento dos dados faltantes foi adotado para não assumir informações erradas sobre sua frequência cardíaca e outros dados relacionados à sua saúde. A coleta de dados foi feita usando uma smartband da empresa Samsung que envia os dados coletados para o aplicativo Samsung Health, de onde foram exportados para serem processados pela arquitetura.

Os dados coletados via smartband são: frequência cardíaca do usuário, pedômetro, nível de estresse e taxas de oxigenação do sangue. Os dados relativos à frequência cardíaca do usuário são divididos em informações úteis, além de sua frequência real. Estes são: o máximo, o mínimo e a data/hora de medição da frequência cardíaca, que é coletada e transferida para o aplicativo em um intervalo de uma hora. Em toda a base de dados, foram analisadas 6.304 ocorrências de medição com valor mínimo registrado de 55bpm e máximo de 164bpm. Além disso, foi identificada uma média de 78,5 bpm nos dados do usuário analisado com desvio padrão de 13,39. Vale ressaltar também que, para uma melhor visualização da distribuição dos valores no banco de dados, os valores dos quartis são: 69bpm para o primeiro quartil, $77 \mathrm{bpm}$ para a mediana e $85 \mathrm{bpm}$ para o terceiro quartil. Ademais, enriquecemos esses dados primitivos calculando a taxa de aumento e a aceleração do aumento da frequência cardíaca para obter mais variáveis que procuram explicar a variação da frequência cardíaca coletada do usuário.

Inicialmente, definimos as variáveis explicativas com base nas derivações feitas e nos dados primários coletados pela smartband. Como a ideia inicial da proposta é predizer o valor da frequência e assim compará-lo com o valor efetivamente medido, foi criada uma nova coluna que contém a frequência posteriormente medida do usuário em questão. Dessa forma, essa nova coluna de informações se tornou a variável dependente do modelo para análise, treinamento e previsão. Usando algoritmos de regressão, podemos comparar os resultados esperados pelas técnicas de aprendizado de máquina (frequência cardíaca prevista) com o valor real coletado (frequência cardíaca real). Assim, é possível verificar se existe uma diferença significativa no intervalo entre esses valores e, assim, apontar um pico de estresse ou ansiedade em relação ao usuário.

A princípio, os algoritmos Support Vector Machines, Decision Trees, and $K$ Nearest Neighbors (KNN) foram usados. Ferramentas fornecidas pela biblioteca Python Scikit-Learn como GridSearchCV também foram empregues para buscar os melhores parâmetros para cada algoritmo e obter o melhor resultado possível deles. Desta forma, parâmetros para cada algoritmo são passados com cada valor que deve ser testado por GridSearchCV. Portanto, uma análise com todas as combinações possíveis de parâmetros é estudada para retornar a melhor combinação encontrada junto com a técnica de validação cruzada para evitar o enviesamento dos resultados. Além disso, também foi desenvolvido 
um trabalho de pré-processamento de dados para dimensionar as informações, além de um trabalho de transformação de dados utilizando a técnica boxcox para trabalhar os dados mais próximos de uma distribuição normal.

Os algoritmos de aprendizado de máquina alcançaram bons resultados, prevendo valores razoáveis. Na grande maioria das previsões, o modelo previu valores em uma faixa de cerca de $10 \mathrm{bpm}$ (batimentos por minuto) para cima e para baixo. Assim, os resultados encontrados para os algoritmos Decision Tree, KNN Regressor, Random Forest Regressor e Gradient Boosting Regressor foram respectivamente: 46 \%, 45 \%, 45 \% e 48 $\%$, usando o Técnica de pontuação $\mathrm{R}^{2}$. Vale salientar que algoritmos como Random Forest e Gradient Boosting conseguiram organizar os dados de uma forma mais bem distribuída quando olhamos para o formato geral em que os pontos são distribuídos ao longo dos eixos. Assim, é interessante notar que estes se destacam como os melhores candidatos para o desenvolvimento do modelo.

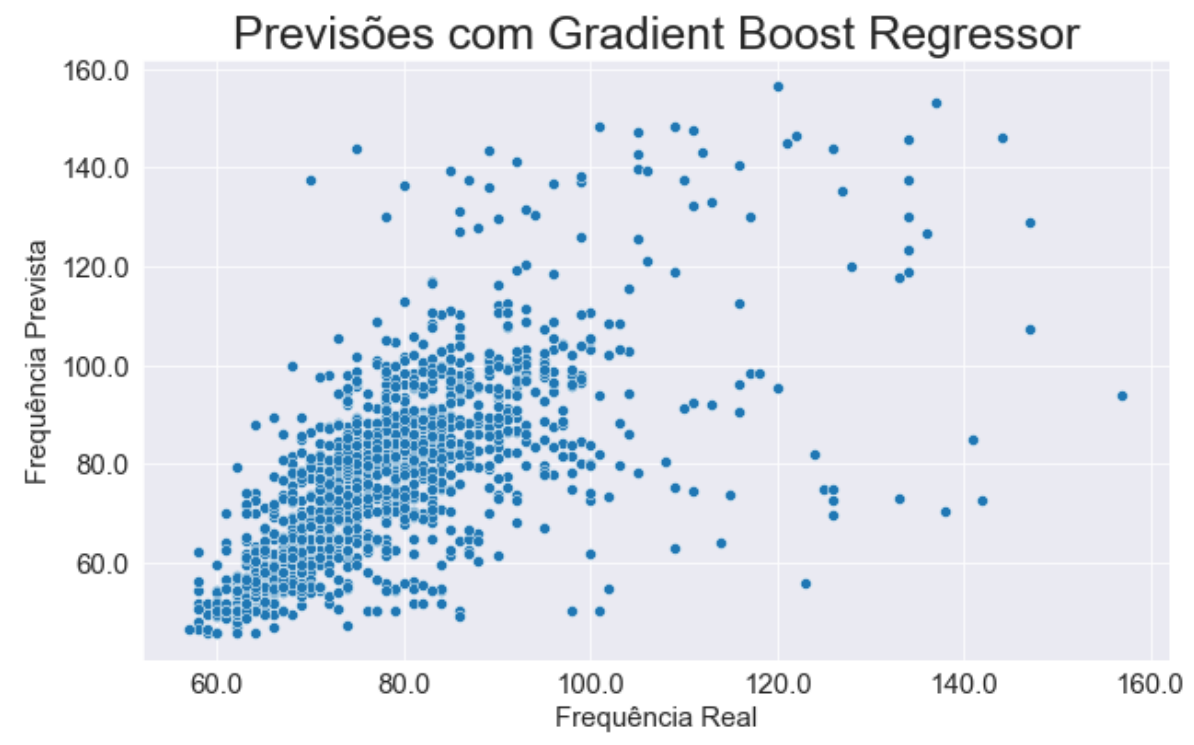

Figura 2. Comparação entre a frequência cardíaca prevista e real

A Figura 2 mostra a frequência cardíaca real e os valores previstos pelo modelo de aprendizado de máquina no algoritmo Gradient Boost Regressor. É interessante notar que pontos próximos da diagonal do plano cartesiano em que o gráfico se encontra representam situações em que os valores preditos pelo algoritmo e os valores de fato notados possuem muita proximidade e, assim, não apontam picos de estresse. Entretanto, pontos distantes dessa diagonal nos mostram casos em que a predição e o que foi de fato visto na mensuração da frequência do usuário possuem grande diferença, indicando que ele está passando por um momento de estresse.

\section{Considerações Finais e Trabalhos Futuros}

Este trabalho apresentou uma proposta capaz de mensurar picos de estresse no cotidiano dos usuários. Foi proposta uma arquitetura para detectar situações de estresse e ansiedade por meio de monitoramento de localização e dados de sensores corporais. Foi realizada uma avaliação com dados reais coletados de um usuário, e seus resultados mostram que as tecnologias e algoritmos utilizados são viáveis e promissores. 
Como trabalhos futuros podemos destacar o uso de dados com granularidade de frequência cardíaca por intervalos de tempo menores, juntamente com informações de mais usuários. Além disso, aplicaremos os dados de localização e de frequência cardíaca em conjunto para realizar análises mais ricas e eficazes. Em conjunto, empregaremos recursos computacionais mais poderosos e modulares para proporcionar maior robustez da arquitetura bem como proporcionar testes com mais algoritmos e mais combinações de parâmetros. Por meio destes, poderemos estudar mais possibilidades para aumentar a qualidade das previsões realizadas a fim de se obter previsões cada vez mais próximas para que os picos de ansiedade sejam mais facilmente notados. Ademais, é interessante estudar a viabilidade e comportamento da arquitetura de forma distribuída ou por meio de parâmetros que representem conexões $W A N$, além de implementar sistemas auxiliares para a gestão de recursos e credenciais.

\section{Referências}

Aditya, S. and Tibarewala, D. (2012). Comparing ann, lda, qda, knn and svm algorithms in classifying relaxed and stressful mental state from two-channel prefrontal eeg data. International Journal of Artificial Intelligence and Soft Computing, 3(2):143-164.

Bove, L. A. (2019). Increasing patient engagement through the use of wearable technology. The Journal for Nurse Practitioners, 15(8):535-539.

Ciabattoni, L., Ferracuti, F., Longhi, S., Pepa, L., Romeo, L., and Verdini, F. (2017). Real-time mental stress detection based on smartwatch. In 2017 IEEE International Conference on Consumer Electronics (ICCE), pages 110-111. IEEE.

Gravina, R. and Fortino, G. (2020). Wearable body sensor networks: State-of-the-art and research directions. IEEE Sensors Journal, 21(11):12511-12522.

Kiran, M., Murphy, P., Monga, I., Dugan, J., and Baveja, S. S. (2015). Lambda architecture for cost-effective batch and speed big data processing. In 2015 IEEE International Conference on Big Data (Big Data), pages 2785-2792. IEEE.

Larcher, L., Ströele, V., Dantas, M., and Bauer, M. (2020). Event-driven framework for detecting unusual patterns in aal environments. In 2020 IEEE 33rd International Symposium on Computer-Based Medical Systems (CBMS), pages 309-314. IEEE.

Munir, A., Kansakar, P., and Khan, S. U. (2017). Ifciot: Integrated fog cloud iot: A novel architectural paradigm for the future internet of things. IEEE Consumer Electronics Magazine, 6(3):74-82.

Priya, A., Garg, S., and Tigga, N. P. (2020). Predicting anxiety, depression and stress in modern life using machine learning algorithms. Procedia Computer Science, 167:1258-1267.

Santhanagopalan, M., Chetty, M., Foale, C., Aryal, S., and Klein, B. (2018). Relevance of frequency of heart-rate peaks as indicator of 'biological'stress level. In International Conference on Neural Information Processing, pages 598-609. Springer.

Wan, J., Al-awlaqi, M. A., Li, M., O'Grady, M., Gu, X., Wang, J., and Cao, N. (2018). Wearable iot enabled real-time health monitoring system. EURASIP Journal on Wireless Communications and Networking, 2018(1):1-10. 